Voix et Images

voixetimages

\title{
Patrick Straram ou la constellation du Bison ravi
}

\section{Claude Gonthier}

Volume 13, numéro 3 (39), printemps 1988

Jack Kerouac et l’imaginaire québécois

URI : https://id.erudit.org/iderudit/200733ar

DOI : https://doi.org/10.7202/200733ar

Aller au sommaire du numéro

\section{Éditeur(s)}

Université du Québec à Montréal

\section{ISSN}

0318-9201 (imprimé)

1705-933X (numérique)

Découvrir la revue

\section{Citer cet article}

Gonthier, C. (1988). Patrick Straram ou la constellation du Bison ravi. Voix et Images, 13(3), 436-458. https://doi.org/10.7202/200733ar d'utilisation que vous pouvez consulter en ligne.

https://apropos.erudit.org/fr/usagers/politique-dutilisation/ 


\title{
Patrick Straram ou la constellation du Bison ravi
}

\author{
par Claude Gonthier, Université du Québec à Montréal
}

\section{1 - L'individu}

Dans les années 70, la contre-culture américaine a été largement diffusée par les médias. Au Québec, contrairement à la France, cette mode a obtenu une large audience. Aussi, la contre-culture américaine a non seulement réussi à modifier le paysage socio-culturel québécois, elle est devenue une pensée, un agir pour de nombreux jeunes intellectuels.

Si une liste exhaustive de ces nouveaux chantres de la contre-culture n'est pas aisée à établir, il est plus facile, en revanche, de déterminer quels sont ceux qui, parmi les intellectuels de l'underground des années 60 (et même fin 50), ont préparé et encouragé l'avènement d'une «contre-culture à la québécoise». L'anthologie Québec Underground désigne à ce titre trois précurseurs fondamentaux: Armand Vaillancourt, Claude Gauvreau, Patrick Straram. Au sujet de Straram, il y est noté

[...] son parti pris à confondre dans ses innombrables critiques vociférantes, sa vie personnelle et l'institution, le fait, le film, l'action à critiquer en une interprétation de la vie quotidienne où se [trouvent] discutés, à sa façon, les concepts du marxisme 1 .

Pour brève qu'elle soit, cette description résume pourtant l'essentiel de la démarche de Patrick Straram, le Bison ravi. Dans le contexte culturel québécois des années 60 et 70, au moment où les intellectuels délivrés du catholicisme se livrent à un rattrapage culturel frénétique, les productions littéraires et critiques de Straram se révèlent des plus intéressantes. Les nouvelles voies esthétiques qu'elles abordent incitent les jeunes intellectuels à s'intéresser aux idées européennes et américaines de la modernité: Bertolt Brecht, Henry Miller, Roland Barthes, Malcolm Lowry, Henri Lefebvre, les Cahiers du cinéma, le rock américain, notamment. A chacun de ces canons de la modernité, Straram emprunte des éléments philosophiques et esthétiques: à Brecht, l'engagement politique de l'œuvre d'art; à Miller, la libération des tabous sexuels; à Barthes, le plaisir et le désir du texte; à Lowry, la légitimité du désir...

1 Collectif, Québec Underground 1962-1972 Tome 1, Montréal, éditions Médiart, mars 1973, p. 24. 
Dans une certaine mesure, de par son savoir étendu et différencié, de par l'audience qu'il a acquis, Straram a influencé les idées et les références de toute une génération d'intellectuels québécois. En outre, sa participation au sein de l'avant-garde des années 60, comme Parti pris ou le Centre D'Art de l'Élysée, a permis une diffusion importante de sa pensée dans les milieux culturels de la «modernité». Pour plusieurs jeunes intellectuels des années 70, un contact personnel avec cet homme a signifié non seulement une ouverture aux textes théoriques et littéraires de la modernité, mais une insertion significative dans les milieux québécois de cette voie littéraire: par exemple, les préfaces de Straram aux textes de Denis Vanier ont, en quelque sorte, cautionné la qualité de cette œuvre auprès du milieu littéraire «moderniste». Les amis écrivains de Straram - et lui-même par retour - se sont donc retrouvés au confluent des nouveaux courants littéraires des années 70.

Dans ce contexte, il n'est pas étonnant de voir Hobo Québec - une des plus importantes revues contre-culturelles des années 70 - rendre hommage à Patrick Straram dans un numéro triple (9-10-11) en 1973. Alors que la contreculture agonise aux États-Unis, celle qui s'est formée au Québec bat son plein et légitime son existence en anoblissant un de ses pères spirituels. La plupart des textes de ce numéro sont des éloges de Straram par de jeunes intellectuels, des amis de longue date, de vieux routiers de l'avant-garde ou de la gauche québécoise qui soulignent maintes fois l'héritage culturel que leur a légué le Bison ravi. Ainsi, Jean-Marc Piotte, un des grands amis de Straram, écrit: Après mes parents [...] Straram fut l'homme qui m'a le plus influencé2. Et Louis Geoffroy abonde dans le même sens: [Dans les années 60] ses textes contribuaient à former le déviationniste global que je suis et m'amenaient à m'intéresser aussi au sort de mes semblables dans cette Alphaville quotidienne où nous vivons ${ }^{3}$.

Straram et la plupart de ses épigones dans ce numéro de Hobo Québec fréquentent les mêmes lieux, gravitent au sein des mêmes réseaux de communication et d'influence; aussi n'est-il pas étonnant que Straram apparaisse ici comme un des catalyseurs culturels du début des années 70 pour ces intellectuels contre-culturels qui font bon ménage avec un certain marxisme.

Mais précisons immédiatement que la contre-culture à laquelle participe Straram n'est pas celle de Mainmise. Si la contre-culture apparaît comme une idée maîtresse de la pensée de Straram, elle se rapproche, avec les nuances idéologiques et culturelles qui s'imposent, de la contre-culture des provos d'Amsterdam et du Do It de Rubin: elle se veut résolument contestataire. Elle rejette presque avec violence la contre-culture dépolitisée que diffuse Mainmise, où prime la recherche d'expériences et de plaisirs individuels devant conduire à une nouvelle perception du monde. Le Bison ravi n'a d'ailleurs pas écrit pour cette revue, toute contre-culturelle qu'elle soit, puisque:

2 Hobo Québec, $n^{\circ}$ s 9-10-11, octobre-novembre 1973, p. 9.

3 Ibid., p. 6. 
[...] la contre-culture américaine da la québécoise telle qu'elle a été, disons, propagée par Mainmise: [ne vaut] rien. [Quand] je dis rien j'exagère: Mainmise a introduit au Québec des valeurs et des notions socio-culturelles de libération fondamentales: l'érigeant en absolu c'était produire un impérialisme totalitaire de plus, qui aliène, qui réifie, qui dé-naturet.

Si la contre-culture de Straram se détache essentiellement de la contre-culture de Mainmise sur la question politique, les deux tendances peuvent par ailleurs engendrer certaines idées et comportements sociaux similaires. Toutefois, chez Straram, l'esprit critique et la volonté politique tendent vers une nouvelle organisation du monde.

Autre différence entre Mainmise et Straram: ce dernier n'a pas de revue ou d' «école» pour diffuser sa pensée contre-culturelle. Mais Straram ne semble pas s'en plaindre. Il compte sur une diffusion messianique de son message, sympathisant avec les intellectuels de divers mouvements de la modernité qui lui assurent une participation à de nombreuses revues underground. Il entre aussi en contact - surtout lors de la grève des réalisateurs de Radio-Canada - avec des artisans de milieux plus officiels. Fondée sur sa participation aux milieux intellectuels et artistiques, la notoriété de Straram ne cessera d'évoluer jusqu'à apparaître, plus tard, déterminante pour l'éclosion de la contre-culture au Québec.

Français d'origine, Straram quitte sa famille à la fin des années 40 . Il mendie et vit alors dans certains quartiers, surtout arabes, de la capitale française; il se lie d'amitié avec des intellectuels marginaux, existentialistes; il fréquente les boîtes de jazz où ils boivent de conserve. La pratique de vie des existentialistes dans ce que Janson appelle «la bâtardise» est, pour une certaine jeunesse intellectuelle française, une volonté de s'opposer à la politique colonialiste et chauviniste de Mitterand. Valorisant les arts et les mœurs qui sont rejetés par la culture et les institutions françaises, elle recherche de nouveaux objets de culte. L'engouement de cette génération pour le jazz n'est pas étranger à ce désir. Arrivé au Québec en 1958, Straram saura profiter de la chance qu'il a eue à Paris, d'entendre les meilleurs musiciens de jazz; en effet, les jeunes intellectuels québécois fétichisent certaines boîtes parisiennes où règne le jazz.

Straram, toujours à l'affût de nouveaux courants de pensées, adhère aussi à l'Internationale Situationnisme. Participant au bouillon culturel qui éclatera en Mai 68, le situationnisme revendique un nouvel ordre social centré sur une critique intégrale de la société contemporaine. Membre de l'équipe dirigée par Yvan Chtcheglov et Guy-Ernest Debord, Straram écrira divers textes pour la revue l'Internationale situationniste où, comme les autres intellectuels du mouvement, il s'oppose à toute pensée égocentrique et mégalomane, que ce soit la philosophie d'Albert Camus ou le mouvement des angry young men anglais. Le situationnisme constitue, en quelque sorte, une «école» pour le jeune 
Straram. Il exprime cette volonté, qui sera toujours celle du Bison ravi, de ne jamais être assimilé à d'anciens courants contestataires récupérés, d'être irréductible à l'autorité (ce que ne sont pas les surréalistes ou la beat generation ${ }^{5}$ ) et aussi de manifester son indignation devant des intellectuels «qui font le jeu du pouvoir».

L'arrivée de Straram en Amérique, afin de se soustraire au service militaire français, est anticipée par une invitation de ses beaux-parents habitant Vancouver. De 1954 à 1958, Straram vivra donc de divers métiers en ColombieBritannique, tout en poursuivant ses lectures (certains de ses amis lui faisant parvenir des livres de France). Il garde ainsi contact avec la production littéraire et philosophique d'avant-garde. À son arrivée au Québec, il conserve ses relations situationnistes dont le prestige lui permet d'approcher les écrivains de l'Hexagone. Avec plusieurs d'entre eux (Miron, Paul-Marie Lapointe, etc.), il publie un Cahier pour un paysage à inventer où sont insérés plusieurs textes situationnistes antérieurement publiés dans l'Internationale situationniste.

Rapidement, Straram conquiert ainsi une place de choix au sein des milieux intellectuels québécois; d'autant plus que sa position situationniste suscite les sarcasmes de critiques officiels qui cherchent manifestement à déprécier cet importun. Or, ces critiques produisent justement ce qu'on attend d'eux. L'édition française de l'Internationale situationniste de décembre $1960\left(n^{\circ} 5\right)$ reproduira même avec joie des extraits critiques de Jacques Godbout et de Jean-Guy Pilon, parus dans le numéro double 9-10 de Liberté à l'été 1960, et de Clément Locquell, dans le Devoir du 16 juillet 1960.

On peut se surprendre de l'association de Straram avec les écrivains de l'Hexagone qui n'étaient ni de grands polémistes ni de grands marginaux. II faut comprendre ici que l'intérêt de Straram pour ce groupe découle de sa connaissance partielle du milieu culturel québécois. Â son arrivée à Montréal, Straram lit tout ce qu'il trouve d'écrivains québécois... à la Bibliothèque municipale! Reconnaissant une certaine «modernité», une certaine revendication sociale aux livres de l'Hexagone, il se tourne d'abord vers ces écrivains. Intégré au groupe, Straram entrera plus facilement en contact avec d'autres sphères littéraires plus marginales, se faisant par ailleurs un nom dans les milieux plus ou moins officiels.

C'est ainsi qu'il travaille à une chronique de critique cinématographique pour Point-de-vue, journal dirigé par Solange Chaput-Rolland! À cette occasion, il rencontre Jean-Paul Ostiguy qui l'engagera pour créer le Centre d'Art de l'Élysée. Secrétaire général, il choisit et présente à l'Élysée bon nombre de films d'auteur boudés par les distributeurs montréalais et, grâce à cette

5 Toutefois, la «beat generation» n'est pas honnie par Straram pour les nouvelles valeurs qu'elle a cherché à promouvoir à ses débuts, mais pour la sclérose petite-bourgeoise où elle a fini par se complaire. Aux yeux du Bison ravi, tout mouvement contestataire a une importance s'il est intègre, car il permet d'éviter certaines erreurs. En d'autres mots, la «beat generation» fut un moindre mal. 
prestigieuse mission pour la diffusion du cinéma d'avant-garde, il voit venir à lui plusieurs intellectuels marginaux. En outre, il se met, suivant son mot, $a$ bien regarder le cinéma, car il découvre le cinéma-critique de Godard, de Varda, de Duras et des Cahiers du cinéma qui modèlent ses options critiques sur le septième art. Par l'avant-gardisme de sa critique, par ses connaissances de première main sur le jazz, Straram devient une figure importante du milieu intellectuel. De plus, connaissant personnellement Chamberland et Major qu'il rencontre souvent au Bistrot, rue Saint-Denis, Straram accepte leur invitation à se rendre au lancement de la revue Parti pris. Ce serait lors de cet événement qu'on lui aurait demandé de signer une chronique de jazz. Straram accepte, mais il abordera aussi d'autres sujets. Il devient même un des membres du comité de lecture des éditions Parti-pris. Fort de cette fonction, il s'oppose à tout geste qu'il juge inconséquent, à tout discours contrevenant à sa lutte, au «conformisme des disours du Pouvoir capitaliste et judéo-chrétien blanc». Il s'oppose ainsi à la publication de Pleure pas, Germaine de Claude Jasmin puisque ce roman ne relève pas d'une étude en profondeur des causes de l'asservissement social du prolétariat. Contrairement à Gérald Godin et à plusieurs autres membres du comité, il ne souffre aucune «concession politique fondamentale».

Pendant quelques temps, Straram occupe aussi le poste de secrétaire de rédaction à Parti pris. Mais bientôt, sa position devient de plus en plus marginale. Ses derniers articles parus dans Parti pris (celui sur Grateful Dead par exemple) annoncent le début d'un intérêt plus marqué pour le mouvement contre-culturel américain. Ils reflètent aussi une certaine distance par rapport aux luttes politiques qui divisent alors la revue. À la même époque, Straram s'inscrit plus que jamais dans le circuit de la presse officielle. Il écrit pour trois publications à large diffusion: Sept-Jours, TV Hebdo et MacLean. Son travail y est nettement moins politique et se borne à de petits «bulletins d'information» sur les films à voir en ville ou au petit écran. Mais déjà ses opinions à l'emporte-pièce font des remous:
À Sept-Jours, au tout début, j'ai écrit un texte, d'ailleurs mémorable puisque j'y consacrais cinq feuillets à Pierrot le fou de Jean-Luc Godard, et quatre lignes à Un homme et une femme de Lelouch, ce qui a fait que pendant les trente premiers numéros de Sept-Jours, j'ai assuré la moitié du courrier des lecteurs ${ }^{6}$.

Il fut renvoyé de Sept-Jours et de TV Hebdo7, et quitta MacLean pour séjourner en Californie de 1968 à 1971. L'expérience de ces magazines le laisse dorénavant perplexe quant à la ligne politique des publications auxquelles il aura

$6 \quad$ Ibid., p. 32.

7 Les «bulletins d'information» des vingt-huit semaines de Straram à TV Hebdo ont été réunis avce une «citation-introduction», quelques notes et des index aux éditions des Herbes rouges sous le titre one + one, cinémarx \& Rolling Stones qui réfêre évidemment à l'œuvre de Godard. 
à participer. Quelques années plus tard, au printemps 1974, il ira jusqu'à démissionner de Hobo Québec lorsque le directeur Claude Robitaille affirme publiquement au Conventum que Les ouvriers, ce sont eux qui polluent! Ce genre d'intervention relève pour Straram d'úne idéologie contre-culturelle petitebourgeoise à laquelle il ne peut adhérer. Notons que c'est durant les années 60 que Straram s'intéresse de façon intensive à la question des autochtones. Désormais, il accompagne sa signature de quatre signes indiens: $1^{\circ}$ deux flèches verticales croisées aux quarts supérieurs, qui représentent la camaraderie, la solidarité; $2^{\circ}$ deux flèches horizontales pointant entre elles deux un point (la conjuration des mauvais esprits); $3^{\circ}$ une ligne horizontale fléchée quatre fois de la gauche vers la droite (la paix): $4^{\circ}$ un point entouré d'un cercle orné à égale distance de quatre séries de trois flèches (le bonheur).

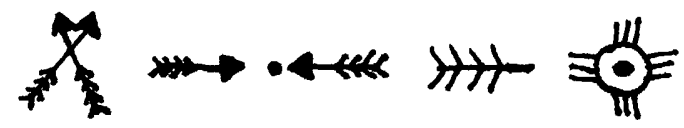

Straram affiche donc une position politique sympathisante aux thèses marxistes. Mais cette position, nous le verrons, se veut dénuée de tout dogmatisme. Selon lui, c'est ce dogmatisme, le marxisme-léninisme sectaire, qui l'obligera à démissionner de Chroniques ${ }^{8}$, car Straram est toujours à la recherche opiniâtre d'une pensée individualiste qui n'accepte aucune direction autoritaire.

De fait, Straram refuse autant le dogmatisme politique que l'apolitisme. À travers le prisme d'une impérieuse individualité, il assume une position politique où les concepts marxistes sont redéfinis à la lumière des influences qui constituent son héritage de vie. Malgré sa sympathie pour les marxistes orthodoxes, reliés à la lutte contre l'ennemi commun (la société capitaliste-fasciste et judéochrétienne blanche), il existe néanmoins une nette distance entre eux. Comme l'écrit Philippe Haeck dans le numéro de Hobo Québec déjà cité:

Dans notre histoire intellectuelle il faut souligner l'activité de P.S.: il est un signaleur: quels films voir (ses chroniques de cinéma ont été publiées par les Herbes rouges), quels disques écouter (jazz, folk-rock), quels textes lire, il est un signaleur passionné, là est sa force mais aussi sa faiblesse. Sa force parce qu'il charge les objets culturels d'une vie, la sienne, sa faiblesse parce que dans sa perspective d'une stratégie révolutionnaire, il faudrait justifier nos choix autrement que par des pulsions individuelles. [...] P.S. ne nous aide pas encore assez d oublier notre individualité en faveur de stratégies collectives, nous ne sommes pas convaincu que l'accent qu'il met sur la vie quotidienne - à la suite d'Henri Lefebvre - soit réellement révolutionnaire [...]9

8 Sur les enjeux au sein de la revie Chroniques, il faudra se reporter à l'article de Pierre Milot à paraître dans un collectif sur la modemité littéraire au Québec.

9 Hobo Québec, loc. cit., p. 7. 
Pour des tenants du discours marxiste orthodoxe - comme Jules Duchastel dans sa thèse de doctorat citée plus bas -, cette notion de l'individualisme libéré et libérateur, régénérant de par sa mission les possibilités d'une nouvelle société moins «fasciste», est le pivot des attaches bourgeoises du discours contreculturel. Elle implique par extension

qu'il existe quelque part, dans un au-deld culturel, la production des formes culturelles qui seraient a un moment rapatriées sous l'effet de la force d'attraction du système dominant. Il y a donc préjugé favorable à l'authenticité révolutionnaire de ces manifestations 10 .

Pour le marxisme, l'individualisme contrevient à une réelle prise de position révolutionnaire. Or, justement, Straram croit à l'inverse que la ligne orthodoxe du marxisme conduit tout droit à l'aliénation:

Les notions d'art bourgeois et d'art prolétarien (comme celles de science bourgeoise et de science prolétarienne) sont non seulement périmées mais aussi contre-révolutionnaires parce qu'elles perpétuent un manichéisme qui ne sert qu'une idéologie, celle du capitalismel impérialisme, et elles sont la marque d'une aliénation qui fausse conscience et action révolutionnaire11.

Afin d'empêcher cette dichotomie aliénante, la pensée du Bison ravi fonde ses articulations sur tout discours, toute ouvre où l'individu n'est pas écrasé par une idéologie autoritaire et dogmatique; le choix de ces discours et de ces œuvres se présentant selon une recherche personnelle que doit étayer un vaste savoir:

L'Opéra de Pékin, oui; à condition que je puisse vivre aussi Janis Joplin et Janis. Se situe là la différence entre monolithisme et dialectique. Aucun politique n'est suffisant qui ne considère fondamental un culturel multiple, à toujours pousser plus avant. Le manque du politique chez Janis importe peu [...] elle a fait éclater un «ordre» culturel sur lequel a besoin de s'appuyer toute esociété bureaucratique de consommation dirigée», comme dit si justement Henri Lefebvre ${ }^{12}$.

En effet, le maître à penser de Straram restera Henri Lefebvre. Dans un sens, la philosophie d'Henri Lefebvre forme même la base des conceptions politiques et esthétiques du Bison ravi.

Prévenu de l'intérêt de la pensée de Lefebvre par les situationnistes vers

10 Jules Duchastel, Théorie ou idéologie de la jeunesse: discours et mouvement social, thèse de doctorat, Université de Montréal, 1974, p. 75.

11 Patrick Straram, Questionnement socra/cri/tique, Montréal, l'Aurore, 1974, p. 108.

12 Patrick Straram, «Le cinéma, bien, mais plus que le cinéma», Chroniques, $n^{\circ} 10$, octobre 1975 . 
1954, Straram n'aurait lu Lefebvre qu'en 1958, lors de son arrivée à Montréal. Mais il parle alors d'une coupure qui infléchi[ra] toute [sa] viel3. À partir de cette date, Lefebvre devient en effet une source importante de la pensée du Bison ravi qui le paraphrase et le cite surtout en ce qui a trait à l'importance du quotidien comme objet de réflexion et d'action dans le cheminement révolutionnaire. Or, la pensée contre-culturelle n'est pas si éloignée de cette révolution au jour le jour. Straram retrouve dans ce nouveau courant des thèmes similaires aux concepts de Lefebvre: accent mis sur l'individualisme, négation et lutte contre l'État dominateur, importance des expériences de la vie quotidienne.

Chez Straram, il semble donc que la contre-culture soit venue ultérieurement se greffer à l'influence de Lefebvre, tout comme ce dernier prolongeait ou enrichissait l'expérience situationniste. La configuration de la pensée de Straram est faite d'accumulations plutôt que de retournements. La vision straramienne se veut élargie, d'où son rapport difficile aux interprétations critiques marxistes qu'il juge réductrices - qu'elles soient d'ordre économiste, historique, scientiste ou qu'elles relèvent d'un «sociologisme léniniste». En vertu de la valeur indiscutable de l'expérience personnelle, Straram exige d'une pensée qu'elle offre la possibilité d'une «utilisation» individualisée. Sa position est en cela semblable à celle de Lefebvre pour qui le marxisme est un outil de transformation, non un carcan idéologique:

Pas de pensée sans u-topie, c'est-d-dire sans vouloir ce qui se découvre en se créant: la différence. Être aliéné, ce n'est pas «devenir autre». C'est se voir et se trouver ramené en arrière, bloqué, empêché d'aller vers le possible [l'autre de la différence $]^{14}$.

Seul le marxisme est un mode d'analyse et de pratique qui inscrit d'abord dans son fondement même qu'il est renouvelable, modifiable, transformable au fur et d̀ mesure que changent mouvement historique et sciences humaines 15 .

Autant pour Lefebvre que pour Straram, la culture est souvent le lieu d'aliénations à dénoncer... ce qui ne va pas sans causer quelques problèmes logiques. Car si la pensée de Lefebvre s'exprime selon un discours proprement philosophique, si elle tire ses arguments et ses réfutations de l'ensemble des propositions de la philosophie française du $\mathrm{XX}^{\mathrm{e}}$ siècle, celle de Straram prend tout discours dénonciateur au pied de la lettre et «l'applique» sans distanciation historique et culturelle à la société québécoise des années 70 . Dire que Janis Joplin brise l'ordre culturel d'une société bureaucratique américaine passe encore, mais décrire la sociêté québécoise comme fasciste relève d'une certaine obscurité conceptuelle.

13 Patrick Straram, Questionnement socra/cri/tique, op. cit., p. 108.

14 Henri Lefebvre, le Manifeste différentialiste, Paris, Gallimard, 1970, p. 178.

15 «Entretien avec Patrick Straram, le Bison ravi», Hobo Québec, op. cit., p. 36. 
Avec l'accumulation des idées qui lui sert de thésaurus personnel, Straram perd le sens des distinctions. Le monde ne semble plus régi par des lois universelles d'historicité et de société alors qu'en fait, il repose tout entier sous le regard du Bison ravi.

La pensée «marxiste» de Straram contient des apories qui sont parfois plus justement le reflet des affinités ou des conflits vécus par l'individu qu'une véritable remise en question des idéologies. Ainsi doit-on comprendre l'accusation anti-contre-culturelle dirigée vers Hobo Québec après que Straram eut quitté le journal:

J'ai fait preuve d'un idéalisme coupable (il y a faute parce qu'il y a idéalisme) en «m'imaginant» 2 ans durant que Claude Robitaille comprendrait a temps qu'un journal, ouvert a tous, ne pouvait devenir qu'ascientifique, lieu d'un brouillard idéologique d'où origine tout fascisme16.

Le fascisme naîtrait-il donc d'un pluralisme des opinions? La solution en ce cas ne serait-elle pas d'écrire pour un journal ou une revue résolument marxiste? Or, c'est en raison de ses divergences sur le dogmatisme rigide des partis ou groupuscules communistes que Straram dit préférer ne pas écrire pour ces publications. Pourtant, à y regarder de près, sa chronique cinématographique «Le cinéma, bien, mais plus que le cinéma» ne pouvait être publiée ailleurs que dans Hobo Québec. Champ libre, la revue québécoise marxiste du cinéma, n'aurait jamais accepté l'individualisme de la pensée straramienne, car Straram penche résolument du côté de la contre-culture qui lui est plus proche:

J'aime infiniment plus écrire pour Hobo Québec que pour Champ libre, même si je trouve que Champ libre va infiniment plus loin. dans un sens que Hobo Québec - et dans l'autre, je trouve que Hobo Québec va beaucoup plus loin 17 .

Ce qui tient Straram à distance du comité de lecture de Champ libre, c'est la ligne politique extrémiste de cette revue de cinéma qui na coïncide pas tout à fait avec les épanchements émotifs de la critique du Bison ravi. Fondement de l'idéologie contre-culturelle, la libre expression de l'individu est essentielle à la pensée straramienne. Tous doivent librement s'exprimer selon ce qu'ils vivent, selon le cheminement de leur individualité. Comment comprendre alors l'accusation de «brouillard idéologique», sinon en dénotant chez Straram une position idéologique parfois moins libertaire qu'il n'y veut parâitre?

Si, pour les contre-culturels de Mainmise, Straram paraît trop politisé, si, pour les marxistes orthodoxes, il est trop réactionnaire, c'est qu'il existe en lui

16 Patrick Straram, Questionnement socra/cri/tique, op. cit., p. 192.

17 «Entretien avec Patrick Straram, le Bison ravi», Hobo Québec, op. cit., p. 35 . 
une possibilité toute personnelle de se constituer un espace culturel où est mis en évidence son rôle de dénonciateur critique. Alors que plusieurs autour de lui abandonnent ou troquent leurs options politiques contre certains avantages sociaux, le Bison ravi revendique, face aux marxistes, la liberté de son cheminement et maintient sa lutte irréductible devant les bourgeois. La lente évolution de son cheminement est la preuve de sa sincérité, soutenue par l'espoir de réaliser un jour son projet non conformiste. À l'encontre de plusieurs jeunes intellectuels qu'il a éveillés aux discours politiques ou esthétiques de la modernité, Straram refuse de sacrifier au «déviationnisme bourgeois et échappe ainsi à toute reconnaissance officielle. Toutefois, cette attitude n'est pas celle d'un renoncement pur et simple. L'absence de reconnaissance institutionnelle est essentielle à l'image sociale du Bison ravi.

Le personnage, en lui-même, est déjà hors de l'ordinaire. Hantant la Cinématèque et les salles de cinéma, trônant à la Casa Española, au restaurant Élégant et aux autres endroits choisis de Montréal avec son cigare et son verre d'alcool, Patrick Straram, le Bison ravi, mène une existence opposée aux institutions et aux dogmes de toutes sortes. Créant autour de lui une aura d'intégrité bien particulière, le personnage revendique une certaine pureté contestataire, certes, mais aussi une singularité qu'il légitime en constituant son univers personnel. Non dénué d'une certaine mégalomanie, il crée son propre monde parallèle: une véritable cosmogonie.

Incarnation de la droiture et de la persévérance, non-conformiste revendicateur et dénonciateur, le Bison ravi peut graviter autour d'institutions dont il n'a cure en définitive. S'il conteste leur hégémonie sur le pouvoir politique et culturel, c'est qu'il existe pour lui un autre monde possible, un monde plus libre, plus sain, où les individus réfléchissent vraiment, où ils ne sont pas réifiés. Ce nouveau monde n'est pas une illusion. La philosophie de Straram n'est pas idéaliste; elle participe d'une forme de matérialisme dialectique. Aussi assure-t-il une existence immédiate à son monde sous la forme non utopique d'une cosmogonie.

L'explication de cette cosmogonie est soudée à la connaissance du fonctionnement de l'individu-existence qu'est Straram: le contact interpersonnel direct en forme la pierre angulaire. D'abord, la cosmogonie édifiée par Straram passe par la rencontre d'individus non réifiés, c'est-à-dire libre des pressions idéologiques de «l'appareil d'État fasciste judéo-chrétien blanc» où évoluent la plupart des exclus. Cette rencontre avec l'individu libre - ou avec une œuvre artistique produite par un individu libre - encourt un jugement de la part du Bison ravi. De là, l'individu peut être rejeté, parce qu'il n'a pas été jugé réellement libre (l'ensemble des rejetés retourne parmi les exclus ou êtres réifiés), ou alors l'individu est accepté au sein de la cosmogonie. Autour de Straram se forme ainsi un groupuscule symbolique, une communauté culturelle où seuls sont admirés - et souvent métamorphosés par l'adjonction de la part de Straram d'un surnom - les individus-amis élus.

Participant à une pensée communautaire, Straram élabore avec les élus une 
dynamique créatrice suscitant diverses manifestations: collectifs d'écriture et de lecture, échanges d'expériences, discussions idéologiques et politiques. Pour Straram, les individus de la cosmogonie vivent en communion astrale avec la constellation des artistes-références. Dans ces conditions, Jack London, né comme [Straram] un 12 janvier sous le signe du Capricorne 18 , et Buster Keaton, né un 4, un 4 octobre comme Jean-Marc Piotte Pio le fou 19 font partie intrinsèque des êtres d'exception de la cosmogonie. Connotée de schizophrénie (état de folie entendu ici comme état positif, voire souhaitable; cf. Prénom Carmen de Godard, lui-même membre pour Straram de «son» univers), la constellation des artistes-références du Bison ravi gravite autour de sa cosmogonie et la magnifie aux yeux de son créateur. Il n'y a donc aucune place pour une quelconque critique venant de l'extérieur. Tout ce qui tenterait de menacer l'équilibre de la constellation est par définition hautement méprisable, de même que ceux qui douteraient de la non-réification de Mozart ou de Janis Joplin...

La fonction de la cosmogonie est d'instituer une possibilité d'existences parallèles unifiées. Touchant à l'ensemble des fonctions vitales de l'intellectuel nouveau, la cosmogonie permet de vivre de façon autosuffisante et de se détacher du sein de la société «fascisante». Pour cette raison, les artistes-références et leurs œuvres seront cités à satiété dans les textes théoriques ou littéraires du Bison ravi puisqu'ils sont essentiels à la re-création de l'atmosphère de la cosmogonie et à sa revendication à l'existence:

C'est pourtant très simple: aucun individu n'est lui-même d'une façon, comme ça, essentiel. C'est encore des mythes catholiques. Un homme est ce que le produit son milieu, sa production, ça implique aussi ses lectures. Or, le peu de sens critique que j'ai aujourd'hui et qui détermine et ma vie quotidienne et ma production d'écritures et mes amours, c'est aussi à travers certaines lectures. Elles ont été tellement bénéfiques pour moi que je crois devoir les mentionner à d'autres. Ça implique aussi $q u$ 'il y a des choses que Bertolt Brecht ou Roland Barthes ou Henri Lefebvre ou ce cher Malcolm Lowry ont dites - j'ai absolument rien de mieux a dire, donc je les cite tel quel20.

Chaque référence élargit la cosmogonie. Créatrices d'une certaine atmosphère, d'un sens pluriel, elles travaillent la singularité. Les choix individuels que reprochent au Bison ravi certains marxistes ne tiennent pas compte de la multiplicité de l'individu cosmogonique. Cet individualisme pluriel engendre un jeu de références aussi bien littéraires que musicales et cinématographiques. De Mozart à Janis Joplin, de Diderot à Keaton, la seule mention d'un nom de la constellation, d'une œuvre significative des artistes-références provoque au sein de la constellation du Bison ravi l'éclatement de toute vision dogmatique.

18 Patrick Straram, Questionnement socra/cri/tique, op. cit., p. 82.

19 Ibid., p. 170.

20 «Entretien avec Patrick Straram, le Bison ravi», Hobo Québec, op. cit., p. 36. 
Sans nécessairement être producteur d'œuvres «signifiantes», tout individu qui partage l'amour des artistes-références de la cosmogonie se donne la possibilité d'être accepté en son sein ou d'être momentanément mieux perçu. Par exemple, puisque Jacques Ferron et Marie-Claire Blais marquent un point d'apothéose et terminal du roman 21 , Claude Jasmin, Jacques Godbout et Victor-Lévy Beaulieu sont stériles et inutiles 22 , sauf si ces derniers s'intéressent à un membre de la constellation sans trop le trahir. Pour cette raison, le livre le plus intéressant [deVictor-Lévy Beaulieu] n'est pas par hasard son essai-poulet sur Jack Kerouac 23. Grâce à une forme littéraire plus avant-gardiste que le genre mort du roman, Beaulieu ne réussit pas trop mal à parler du héros de la «beat genaration». Mais Straram se réserve aussi le droit de rejeter ceux qui trahissent le projet, se laissant séduire par les attraits matériels du capitalisme, ceux dont les ouvres portent inévitablement les stigmates de cette tare. C'est donc sans surprise qu'on découvre chez Straram un flot ininterrompu d'anathèmes et de diatribes sur les artistes-symboles de l'Autorité et de l'Ignorance. Pour Straram, la plupart des humains de la société capitaliste sont comme les habitants de la ville menacée de son roman la Faim de l'énigme: ils ne savent pas comment améliorer leur sort puisqu'ils ne réfléchissent plus. Et, comme dans ce roman (nous reviendrons un peu plus loin sur cet unique roman du Bison ravi), la solution ne peut provenir que d'un outsider. Si l'isolement résultant de la cosmogonie conduit évidemment au désespoir, c'est un désespoir assumé, fier et hautain. Vivre au sein de la cosmogonie donne un tel dynamisme, un tel sentiment de vie réelle et ressentie qu'il ne peut y avoir de déception.

Observons maintenant l'hésitation où se trouve Straram d'accepter des œuvres populaires en raison de la lutte à la société capitaliste fasciste. En effet, la popularité d'une œuvre devient souvent suspecte pour les intellectuels et doit presque nécessairement conduire à son rejet, car il convient qu'une distance esthétique nette sépare les deux mondes:

\section{[...] pour moi dans Boris [Vian] il y a une critique, qui est négligée aujourd'hui; la lecture de Boris aujourd'hui est complètement superfi- cielle, et ça c'est parce qu'il est devenu une mode [...] Boris Vian, [c'est] huit pages dans Paris-Match24.}

La seule façon d'accepter une telle cuvre serait d'en faire une lecture fondamentalement «autre», car la mode est cause d'abrutissement; on ne saurait y souscrire. De même, une large diffusion d'œuvres «dérangeantes» concourt à une lecture superficielle qui trahit nécessairement les intentions de l'auteur. Opposée à cette lecture superficielle accomplie par des rejetons de l'Autorité, se situe «l'autre lecture» vraiment critique qui met en évidence le piège de la réification

21 Patrick Straram, Questionnement socra/cri/tique, op. cit., p. 45.

22 Ibid, p. 45.

23 Ibid., p. 45.

24 «Entretien avec Patrick Straram, le Bison ravi», Hobo Québec, op. cit., p. 31 . 
où nous entraînerait une société capitaliste/fasciste. Certaines œuvres, bien que populaires, pourront donc être admirées parce que l'autre lecture y trouvera des valeurs différentes de celles de la critique officielle. C'est le cas du Dernier Tango à Paris où Straram lit un regard lucide sur la décadence des rapports humains au sein de la société fasciste, alors que d'autres n'y voient qu'une étude de mœurs «profonde» beurrée de quelques détails piquants!

Mais Straram penche plutôt vers la recherche et la valorisation de productions d'artistes peu ou mal connu(e)s dont les pensées et les actions sont marginales. D'où l'importance de son rôle d'informateur:

À l'époque, j'étais un peu dans la situation du gars qui est seul à les connâtre [Vian, Vailland], qui essaie de les faire connaître, et à partir du moment ou ils deviennent mode, ton travail est terminé, tu passes a autre chose, tu laisses tomber 25 .

On remarque combien la démarcation opérée par ce rôle de «pédagogue social» est fortement imprégnée d'une condescendance ironique face aux autres intellectuels:

Je n'ai pas renoncé à Boris Vian puisque je m'appelle le Bison ravi, ce qui est un emprunt direct à Boris Vian dans son roman Trouble dans les Andains, ce que je me fais toujours un plaisir de mentionner puisque tous ceux qui ne jurent que par Boris Vian ne savent même pas qu'il a écrit Trouble dans les Andains 26.

La constellation des artistes-références est mobile, ouverte et changeante: elle se fait une loi de découvrir de nouveaux «vrais» créateurs. Cette recherche de nouvelles étoiles est tout aussi nécessaire que l'exploration de celles déjà connues. Suivant les théories orientalistes d'Alan Watts, penseur de la contreculture américaine, la vie de la cosmogonie doit ainsi participer à une perpétuelle confrontation de ses propres valeurs. Le Yin et le Yang se succèdent: de nouveaux artistes viennent se substituer aux étoiles mortes. Le choix des «stars» répond de cette façon à toute accusation de sclérose, puisqu'à la limite, le dogme pernicieux peut toujours refaire surface, dangereusement soutenu par une individualité despotique. Aussi, la cosmogonie du Bison ravi contourne l'épineux problème en instaurant la toute-puissance d'un comportement destructeur et fondamental: la critique. Par celle-ci, Straram entend éviter l'institutionnalisation de son univers. Si Hobo Québec anoblit Patrick Straram, c'est en tant que monument de la contre-culture québécoise. Bien que non consacré par une institution officielle - ce qui constituerait aux yeux de Straram une collusion avec le pouvoir - le Bison ravi reçoit de la part d'une institution underground une légitimation de son statut privilégié (sinon pur) au sein des groupes engagés de la modernité. La critique des institutions et de la société capitaliste relève alors d'une institutionnalisation parallele... 
Sûr de l'indépendance de son discours critique face à toute institutionnalisation, Straram est amené à ironiser sur les diffuseurs officiels:

C'est un scandale que Radio-Canada ne me confie pas une heure ou deux d'antenne chaque semaine pour y commenter les musiques de mon choix. Scandale il y a, mais c'est un scandale inhérent à la politique d'ignorantisation de Radio-Canada, dont tous les programmes sont conçus pour insignifier les auditeurs 27.

Toutefois, ces contradictions de la pensée straramienne, assumées selon lui, constituent la preuve d'une constante évolution/révolution de sa critique, moteur d'une «démarche dialectique» qui cherche à réaliser un être détaché de tout «impérialisme idéologique»:

Je relis dans ce que j'ai écrit il y a seulement trois ans des trucs qui me font bondir. Je trouve ça lamentable. Mais si je n'avais pas écrit Irish Coffees, je ne serais pas capable aujourd'hui de trouver ça lamentable. J'en suis à une époque ou je tente de parler d'amour, de politique ou d'écritures, ou de parler avec vous, selon une pensée marxiste-léniniste tout en n'endommageant absolument pas cette espèce de romantisme ou de lyrisme, ce que j'appelle style de vie, parce que pour moi la vie quotidienne est aussi importante que la théorie. Mais je peux pas arriver a concilier tout, tout de suite. Ça prend du temps 28.

\section{2 - Critique et textes littéraires}

Pour éviter le totalitarisme, aussi bien que l'apolitisme, la critique straramienne pluralise le discours:

Si on allait très très loin comme ça en fouillant qui je suis, on verrait que je suis a la fois Boris et Barthes, et que l'un sans l'autre ne m'intéresse pas. Et ça, ça rejoint la Bâtardise, la dialectique. Donc une seule pratique de vie: la critique 29 .

Chez Straram, les œuvres des artistes-références de sa constellation sont incorporées à son propre discours pour le pluraliser. De cette fusion naissent des contradictions nécessaires à l'effritement du discours univoque. Les références les plus hétéroclites peuvent donc se retrouver sur une même page, sur une même ligne - elles sont même souhaitables - puisqu'elles assurent le pluralisme du discours. Il ne faut donc plus concevoir les divers discours cités comme des entités négativement antagonistes. Au contraire, leur participation au discours de

27 Patrick, Straram, «Une journée dans la vie de camarades», Portraits de voyage, Montréal, l'Aurore, 1975, p. 90.

28 «Entretien avec Patrick Straram, le Bison ravi», Hobo Québec, op. cit., p. 34.

29 Ibid., p. 33. 
Straram, tout comme à l'atmosphère de la cosmogonie, assure un dynamisme philosophique et politique essentiel. Comme chez Deleuze, le sens n'est plus causal, mais sériel:

Je prends a la lettre ce qu'écrit Roland Barthes lorsqu'il écrit que «la CRITIQUE, la déchirure des enveloppes idéologiques dont notre société entoure le savoir, les sentiments, les conduites, les valeurs est le grand travail du siècle». Parce que nous vivons au Québec un état fasciste qu'accentue une réaction de plus en plus prompte d défaire tout ce qui est critique, quand la gauche néglige de plus en plus d'intervenir dans le champ cultureß3.

La critique, omniprésente dans la pensée du Bison ravi, est aussi une base d'existence et d'écriture. Tous ses écrits en portent la marque: textes théoriques, chroniques cinématographiques, œuvres littéraires... La distinction entre genres littéraires chez Straram est donc parfois bien aléatoire et leur imbrication, courante. Plus intense, plus achevée que la conversation, l'écriture est le langage déterminant qui permet de tracer le moment de l'Histoire où l'existence se vit. Elle valide l'existence de la cosmogonie, la réinvestit du plaisir de la recherche, de la curiosité nécessaire à sa vitalité:

Pourquoi résister au plaisir, ce travail (l'entendrons comme «le métier, cet étrange réconfort» de Malcolm Lowry), de pousser plus avant l'investigation, de m'investir plus avant dans le champ de l'écriture, auquel à la fois je suis réductible et qui m'illumine - l'écriture valant d'abord pour moi par le devenir de sens qu'elle ajoute au vivre?31

Cette conception de la valeur de l'écriture suscite dans les écrits straramiens la présence de trois thèmes principaux:

1) la relation au quotidien: les faits et gestes, rencontres et conversations «signifiantes» avec les élus;

2) la critique, par dénonciation ou encensement, de tout ce qui entoure, de tout ce qui investit le quotidien et en particulier les œuvres artistiques vues, lues, ressenties;

3) la poésie des sensations perçues et vécues lors des deux états précédents: l'écriture exprimant ici le sentiment ressenti lors de l'événement et qui ne peut s'exprimer que par l'écriture.

Pour démontrer la relation étroite entre l'écriture de Straram, sa vie et les amis qui forment sa cosmogonie, nous donnerons ici un long extrait d'un récit

$30 \quad$ Ibid., p. 31.

31 Patrick Straram, «Une écriture critique une érotique/politique», Chroniques, $\mathrm{n}^{\circ} 6 / 7$, juin/juillet 1975 , p. 98. 
intitulé «Une journée dans la vie de camarades», tiré d'un collectif, Portraits de voyage, dont les autres textes sont signés Jean-Marc Piotte et Madeleine Gagnon:

Le téléphone sonne. C'est la gentille Lionne. «Il se prend pour un loup. Il me dit que c'est la fin du monde. J'entends Pio hurler. La Lionne lui passe le téléphone, je lui parle, il ne répond pas, il hurle. Je dis à la Lionne que j'arrive. Je m'habille. Après des mois de recherche, pour l'offrir à LII.A.M.A4, je viens de lire l'admirable le Loup des steppes de Herman Hesse, que m'avait révélé Jean-Claude Guilbert le Berger en 1952. Je suis donc en territoire connu. Je téléphone à Lorenzo Morin, à M.M., à l'Hôpital Sainte-Jeanne d'Arc, à l'Hôpital Saint-Luc, personne ne me fournit de renseignements, mais l'Hôpital Saint-Luc me dit qu'il faut appeler la police pour obtenir qu'une ambulance vienne chercher Pio le fou. je téléphone à la gentille Lionne. «Il s'est jeté à travers la porte vitrée. Il est en sang. J'ai appelé le Toucan maybe, il vient de descendre.» Au coin des avenues de l'Esplanade et Laurier j'attends en vain un taxi, dans la grande froidure à rafales de vents qui font crisser les bancs de neige. Je marche jusqu'à l'avenue du Parc, j'attends. Enfin un taxi. Il n'y a personne chez Madeleine et Piotte, la porte est brisée, des éclats de verre partout, des flaques de sang rougissent la neige. Je sonne à côté chez Patricia et Derek, pas de réponse. J'attends rue du Parc Lafontaine qu'un taxi passe. La gentille Lionne et le Toucan maybe ont dû conduire Pio le fou à l'hôpital, je vais retourner chez moi attendre que l'un ou l'autre m'appelle. Taxi. Je bois un autre nescafé. Le téléphone sonne. C'est le Toucan maybe: «We are at the Notre-Dame Hospital. Could you come, it'll be better for Madeleine?» «Sure. Coming right away.» J'attends un taxi, dans la grande froidure à rafales de vent qui font crisser les bancs de neige. Hôpital Notre-Dame. Urgence. La gentille Lionne et le Toucan maybe. Il est 3 heures 30. Pio le fou est entre les mains des docteurs, ils lui ont donné quelque chose pour dormir, il se démenait et gueulait comme un cochon qu'on égorge quand il est arrivé, c'est Derek qui a couru au poste 17, Pio s'était soudain calmé quand il avait vu le Toucan maybe, il est resté très calme quand il est revenu avec les policiers, c'est à l'hôpital qu'il a refait une crise, il a perdu énormément de sang, les docteurs lui font des points de suture, ce sont les pieds qu'il s'est coupé surtout. Le sexe? Rien. Heureusement. Il avait juste un poncho. La gentille Lionne est crispée, la fatigue par l'intérieur dévasté accentue la douceur de sa beauté calme $e^{32}$.

Cet extrait permet de constater les liens étroits et fraternels qui unissent les différents membres de la cosmogonie du Bison ravi. Une bonne part de l'œuvre rend ainsi compte de leur existence, en prose comme en poésie. Quand Straram encense une des auvres de sa constellation, son lyrisme est alors inépuisable et

32 Patrick, Straram, «Une journée dans la vie de camarades», loc. cit., p. 66. 
luxuriant, comme dans cet extrait d'une de ses envolées à propos de l'Adagio en si mineur K.540 de Mozart:

Et alors, c'est la rentrée des trois thèmes qui vont successivement quitter la zone d'ombre. Le tragique sujet en ré mineur, présenté maintenant en sol mineur, chante, douloureusement encore, mais empreint d'une grande douceur. Le $3^{e}$ sujet peut apporter à présent son cantabile resurgi de la vingtième année. Voici enfin la modulation en sol majeur, voici les premières caresses du soleil. Les trilles, naguère tumultueux, ne sont plus que le délicat remous d'alouettes matineuses, plane la ligne impondérable du premier violon 33 .

Ces seuls exemples pourraient faire croire que Straram n'est qu'un esthète du quotidien. Mais cette écriture suppose aussi un engagement intégral et immédiat de l'écrivain afin de pratiquer politiquement un matérialisme de la tendresse ${ }^{34}$. Si les textes sur la quotidienneté et les rencontres avec les acteurs de la cosmogonie sont des témoignages d'actualité, ils doivent le plus souvent s'appuyer sur des passages concernant les supercheries du Pouvoir, car

Aujourd'hui, Québec. La bâtardise accrue, de me rcouper», en apparence (qui suffit aux autres, que je me déculpabilise plus), d'une consumation fondement du sens qu'a ma vie, accru ce sens d'INJUSTICE, INCONSISTANCE et INDIFFÉRENCE (motivant la critique radicale sens de ma production) par l'absence dans sa présence de la femme aimée, qu'insistent mon hypersensibilité et mon intuition d'un nouveau romantisme indispensable (style, de vie dans la quotidiennetél productions, dont la «mise en pratique» se joue d'abord dans la matérialisation ou non de l'amour ( camaraderie). Métis et fleur bleue 35 .

Les textes de Straram, ancrés dans l'actualité, renient les notions bourgeoises de l'Art Immatériel, Intemporel, Éternel. La pertinence du texte straramien se trouve dans la justesse de frappe de son discours politique et dans l'expression d'une nouvelle sensibilité.

Peu d'œuvres du Bison ravi different de cette optique. Les textes expriment tous les leitmotive de la philosophie politico-existentielle du Bison ravi; les mêmes phénomènes, les mêmes films, les mêmes livres reçoivent d'un texte à l'autre les mêmes anathèmes, les mêmes encensements. Et toujours la critique de la production culturelle, délaissant le plan de l'expression, s'attache à celui du contenu.

Le [mauvais] film comblera la mauvaise foi de publics en leur donnant bonne conscience, tout en insinuant qu'ils osent être bien plus

33 Patrick Straram, Questionnement socra/cri/tique, op. cit., p. 258.

34 Ibid., p. 154.

35 Ibid., p. 31. 
«intelligents», des publics qui ne fourniront pas d'hommes pour les luttes ouvrières réelles, la production d'un Comité d'Information Politique l'envers, identique, d'un catéchisme dont l'individu garantit l'«humanitaire», le lieu d'une ignorance d'un langage et d'un idéalisme qui vont effectivement de pair 36 .

Le cinéma véritablement révolutionnaire est celui qui questionne son propre procès de production, par rapport à des règles édictées pour reconduire l'idéologie de la classe possédante / dominante, tout en questionnant sur ses conditions d'existence dans le rapport des forces actuels [sic] un spectateur qu'il veut transformer en producteur (relire polémique Marx / Weitling et théâtre et textes polémiques de Brecht), le cinéma de Jean-Luc Godard, de Jean-Marie Straub, de Jacques Rivette, de Gilles Groulx le Lynx inquiet, est-il extrêmement difficile à lire pour le moment, et alors qu'on ne lit pas encore ce qu'ont de révolutionnaire le cinéma de Dziga Vertov et aussi bien celui de Buster Keaton, et celui de tant d'autres, un peu vite "catalogués» à l'intérieur des limites bourgeoises qu'ils enfreignaient plus réellement et profondément que tant de militants zélés à l'idéalisme leur interdisant un savoir critique suffisant du cinéma possible... 37

L'influence des Cahiers du cinéma est très nette dans cette référence à Vertov, que Godard - qui admirait son cinéma-vérité et son montage hachuré a contribué à faire connaître.

Si le Bison ravi fait office d'iconoclaste dans la plupart de ses textes, c'est qu'il se veut particulièrement attentif à tous événements ou productions culturels prétendant à la modernité. Aucun d'eux ne voit le jour sans que Straram ne s'interroge et n'exprime sur lui un jugement tranchant. Le plus souvent sont ainsi dénoncées comme pseudo-modernes des productions relevant d'un organisme officiel qui ne peut que favoriser, selon Straram, des positions apolitiques ou faussement progressistes. À l'inverse, Straram encense les productions intellectuelles appartenant à sa cosmogonie et dénonce l'inaccessibilité de ces œuvres au grand public. Il exprime combien la censure dont ils sont victimes s'exerce d'abord en raison du message critique véhiculé.

Chez Straram, la critique est donc le lieu d'une dichotomie peu dialectique: les «bonnes œuvres» d'un côté, les «mauvaises» de l'autre. Et les raisons pour lesquelles elles sont ainsi étiquetées demeurent souvent très floues, voire énigmatiques. Par exemple, la critique straramienne ne définit qu'accessoirement les éléments fautifs d'une œuvre rejetée. Elle ne parle qu'au niveau du discours. Elle ne livre que l'aboutissement de sa réflexion et donne en bloc son jugement.

Midnight Cowboy de John Schleisinger: la manipulation a fins d'occultation - le cinéma reflet policier de l'idéologie impérialiste qu'il 
reconduit - est d'autant plus écourante que son grotesque n'est pas détecté, le public se complaisant dans le mensonge qu'on lui impose 38.

En raison de la valeur négligeable des «mauvaises œuvres», Straram les juge souvent de façon expéditive. En revanche, les «bonnes œuvres» ne cesseiont de produire un flot ininterrompu de relectures, parfois éminemment semblables. Or, ces répétitions sont conscientes. Elles revalorisent, comme nous l'avons déjà dit, la cosmogonie par la mention des plus beaux fleurons de la constellation et participent à la fonction didactique qu'entend assumer l'auteur.

Certaines choses sont à répéter constamment. Répétition: transgression du linéaire des pouvoirs. Il y a certaines choses qu'on ne répétera jamais trop, c'est certain. Si on les répétait vraiment, Dupuis et Bou-Bou n'existeraient pas aujourd'hui, précisément. Et quand je dis Dupuis et Bou-Bou, ça veut dire un état néo-fasciste du Québec qui va s'accentuant 39 .

Straram utilise aussi la répétitivité comme forme esthétique dans sa poésie. Ces répétitions ont pour objet d'investir le quotidien d'un constant retour sur luimême, mais aussi de souligner son envoûtement. Chez Straram, la répétition a la valeur à la fois d'un mantra et d'un rondeau musical. Un bon exemple apparaît dans la Faim de l'énigme où les scènes de torture sont reprises mot à mot, soulignant l'inéluctable inhumanité de ceux qui détiennent le Pouvoir. Ce roman constitue à n'en pas douter une exception notable dans l'œuvre du Bison ravi. En regard des textes poétiques, l'argument en est relativement simple: deux messagers du Gouvemeurs arrivent un jour avec une troupe d'hommes à tête de sable. Ils demeureront dans la ville pendant vingt-deux jours et vingt-deux mois puis mettront à mort tous les habitants à moins que l'un d'eux ne réponde à l'énigme du Livre des Onze Ponts et des Onze Lacs. Chaque habitant n'a qu'une chance de répondre à l'énigme; s'il échoue, il est découpé à la scie ronde. Un habitant qui tente de s'évader est condamné à se faire scier la plante des pieds. Le spectacle de ces tortures est obligatoire pour tout habitant. Nouant intrigues amoureuses ou fraternelles, des marginaux comme Zamco le Métis, Leiris, Koçoi, le Borgne, le Bateleur qui fréquentent la Taverne du Bison et la belle Alanis entreprennent de résoudre l'énigme. La quête devient ésotérique. Zamco y perd la vie, mais permet ainsi à Leiris de dévoiler la solution. Celui-ci ne le fera qu'à certaines conditions:

Que les policiers deviennent des ouvriers. Que le Borgne et toi le Bateleur soyez les enseignants des enfants. Qu'on me donne la Loterie, le Bateleur, où ce ne sera plus pour de l'argent qu'on jouera, où je ferai jouer de façon à ce qu'il y ait pour tous questionnement du jeu, comme un autre enseignement avec tous. Que la Mère propose aux deux Messagers du Gouverneur de travailler avec elle d̀ la Taverne du Bison.

38 Ibid., p. 169.

39 «Entretien avec Patrick Straram, le Bison ravi», Hobo Québec, op. cit., p. 34. 
Qu'Alanis s'occupe de l'Hôpital et de fêtes. Et que chaque année la population discute pour décider qui sera responsable de quoi. Il faudra aussi que nous choisissions d'être de la plus extrême vigilance et de combattre sans répit tous ceux qui n'accepteront pas que nous vivions un tel kétat» autre, ayant compris pourquoi, qu'ils nous attaquent et soumettent à leurs lois ou qu'ils tentent de nous acheter 40 .

Le projet utopiste n'est pas la moindre originalité de ce roman pour les lettres québécoises. Une connaissance approfondie de l'œuvre du Bison ravi permet de rapprocher la Faim de l'énigme de sa production courante. Le roman renvoie tout entier aux trois thèmes chers à la cosmogonie straramienne: récit de la vie quotidienne de marginaux; réflexions politiques; envolées poétiques sur la vie. Toutefois, à l'encontre de certains textes parfois verbeux, la particularité de ce roman est d'avoir réussi à maintenir à flot la dimension dramatique. L'auteur le doit probablement en grande partie à son inspirateur.

À partir d'un livre sur la pensée chinoise de Genet [...] j'ai écrit la Faim de l'énigme, conte construit autour d'un certain nombre de schémas relevés par Genet ${ }^{41}$.

Cet orientalisme occidentalisé que nous retrouvons à l'occasion chez Straram donne au roman un aspect onirique se rapprochant des œuvres de Boris Vian. La cosmogonie du Bison ravi n'en est que mieux représentée et, par là, Straram expose son rêve utopique, sans les frasques des anathèmes et des diatribes conceptuelles. Le roman laisse voir plus clairement combien Straram est avant tout un poète à la recherche d'un monde meilleur:

Il est très vrai que ce qui me frappe le plus, c'est: l'Injustice - ça je peux en traiter en termes marxistes et je crois que j'essaie de le faire -. mais aussi l'Inconstance et l'Indifférence. [...] Je vis dans un monde ou la réification des individus, conformément a la logique d'un système capitaliste qui au préalable est catholique puisque l'un engendre l'autre, me fait éprouver un mal absolument écœurant de voir des êtres qui ne le sont plus $[\ldots]^{42}$.

L'utopie de la Faim de l'énigme est bien ce projet fondamental qui soutient la cosmogonie straramienne. Dans le roman, la population accepte cette nouvelle société, mais, pour le Bison ravi, dans la vie réelle, le processus conduisant à l'autonomie et à la liberté est plus ardu. La toute-puissance de l'État sur les individus brime leur possibilité d'émancipation. Patrick Straram ne conçoit pas y être lui-même parvenu. Il est conscient du fait que son vocabulaire et, en particulier ses adjectifs privilégiés (magistral, fondamental, essentiel, primordial)

40 Patrick Straram, la Faim de l'énigme, Montréal, l'Aurore, 1965, p. 165.

41 «Entretien avec Patrick Straram, le Bison ravi», Hobo Québec, op. cit., p. 29.

42 Ibid., p. 34. 
relèvent plus de la théologie que du marxisme. Mais il revendique ses erreurs comme de nécessaires ajustements dans l'évolution de sa pensée. En un sens, Straram, en faisant constamment état de l'inachèvement de sa démarche, va au devant de la critique.

De même, pour lui, chaque œuvre littéraire ou théorique doit être perçue comme un jalon signalant simplement le cheminement parcouru; toute tentative de lecture est jugée inapte à saisir «l'intégral de lecture» puisqu'un texte est un instant de vie clos sur lui-même et entièrement dépendant de son historicité.

Dans ces conditions, il n'est pas étonnant que le Bison ravi demande à ce que la lecture de ses «écritures» ne soit pas effectuée sans une bonne connaissance des auteurs-références de sa constellation, ou encore, sans une écoute, de la part du lecteur, de musique non réifiée:

Comprendre que je cherche à désigner des signes matérialistes que me suggèrent des matières sonores, allant dans le sens de mes préoccupations qui donnent un sens à ma vie: le questionnement du matérialisme dialectique. [...] (Une lecture véritable de l'intégral de l'écriture demande ici l'écoute au fur et à mesure des disques cités. $)^{43}$

La lecture ne peut être qu'un parcours de la pensée plurivoque du Bison ravi. Pour s'y engager, il faut un acte de foi de la part du lecteur, un réel effort de "communion». Cette conception de la lecture renvoie à une certaine forme de messianisme spirituel, car le moindre détachement suscite, selon Straram, des difficultés insurmontables à la compréhension de son monde. Or, pratiquement, si la musique n'aide pas le lecteur à recréer l'atmosphère de communion souhaitée, que doit-il faire? Se percevoir comme définitivement rejeté de la cosmogonie?

Tout en croyant à la pluralité de l'écrit et de ses lectures, Straram tente dans un même souffle de recrêer une certaine «objectivitê» de la lecture: seuls les élus peuvent véritablement saisir son message. En conséquence, c'est un acte de foi qu'impose le texte straramien à tout lecteur éventuel et la «conversion» de ce dernier résume et sanctifie le but et la nécessité de l'écriture: l'élargissement de la cosmogonie et le rayonnement de la constellation du Bison ravi.

Cette mission de l'écriture est indissociable d'une certaine volonté du Bison ravi de demeurer obscur. L'indéchiffrabilité est une nécessité puisque le sens ne doit être transparent qu'aux fidèles. Aussi, lorsque des amis de Straram lui suggèrent

que trop de jeux de mots [...], de noms, de sur-noms, d'associations d'idées, d'images de situations, d'insensées références [ne sont] intelligibles qu'à quelques-uns, [et risquent] d'imposer au lecteur non prévenu,

43 Patrick Straram, Questionnement socra/cri/tique, op. cit., p. 182. 
celui auquel s'adresser d'abord, des espaces-temps d'un hermétisme irritant, d'une obscurité exaspérante 44 ,

l'ironie de Straram répond:

Outre qu'une connaissance de toutes les écritures fasse probablement lisibles tous ceux-ci [les membres de la cosmogonie], je tiens les sousentendus qu'il peut y avoir dans une écriture pour parties intégrantes de celle-ci, points, évènements [sic] qui échappent à un entendement premier mais, le camouflage alertant, aussi révèlent des intensités ou des nomenclatures faisant tel écrivain et telle son écriture 45 .

La fierté d'écrivain libre de Straram n'est pas dénuée d'une condescendance nécessaire à sa position de dénonciateur rejeté par la culture officielle.

Être modeme et progressiste implique la recherche d'un nouveau langage pour mieux exprimer la perception d'un monde nouveau. Ces horizons tout neufs ne sont pas sans évoquer Foucault, Derrida et Deleuze. C'est d'ailleurs avec une citation de ce demier qui résume très bien le monde straramien que le Bison ravi ouvre ses Quatre Quatuors en trains qu'amour advienne:

Quand on travaille, on est forcément dans une solitude absolue. On ne peut pas faire école, ni faire partie d'une école. Il n'y a de travail que noir, et clandestin. Seulement c'est une solitude extrêmement peuplée. Non pas peuplée de rêves, de fantasmes ni de projets, mais de rencontres. Une rencontre, c'est peut-être la même chose qu'un devenir ou des noces. C'est du fond de cette solitude qu'on peut faire n'importe quelle rencontre. (Gilles Deleuze «Dialogue» (avec Claire Parnet) (1977) collection «Dialogues» éditions Flammarion 46.

L'écriture de Patrick Straram relève parfois d'un orientalisme très contreculturel par son association imperturbable des contraires. Straram emprunte aussi à des philosophes français tel Deleuze. Ces influences reflètent toutes deux cette volonté de fléchir la ligne autoritaire et rigide du Pouvoir. Constamment, farouchement, elles favorisent une attitude fraternelle envers le rebelle: le désir d'être autre, d'être différent. Un témoignage de la scuur de Patrick Straram donne à entendre que dès l'adolescence, Straram possédait cette propension à croire aux valeurs de la marginalité:

Il faisait entièrement ce qu'il voulait, ce qu'il choisissait. Il ne se laissait pas faire [...]. Je l'ai toujours connu n'en faisant qu'à sa tête, sans

44 Patrick Straram, Irish coffees au no name bar \& vin rouge valley of the moon, Montréal, l'Hexagone/l'Obscène Nyctalope, 1972, p. 7.

45 Ibid., p. 7.

46 Patrick Straram, Quatre Quatuors en trains qu'amour advienne, Montréal, éditions du Noroît, 1984, p. 11. 
crainte des dangers, pourtant largement prévenu contre eux par mes parents. Il avait des amis extraordinaires [...] $]^{47}$.

Éternel contrevenant à la Loi, voire à la sienne propre, Patrick Straram s'est particulièrement attaché à faire de sa vie une illustration de ses écrits. Il est par là l'un des rares écrivains québécois à avoir été conséquent avec ce qu'il prônait. Idéologiquement attaché à la contre-culture et au marxisme, vivant pour une modernité toujours renouvelée et autocritique, il demeure, pour toutes ces raisons, un élément important des milieux intellectuels des années 60 et 70.

Publiés dans les années 80, ses Quatre Quatuors en trains qu'amour advienne, malgré une synthèse intéressante de tous les éléments de son écriture, n'obtiennent pas l'audience qu'ils méritent auprès du milieu. C'est l'heure des remises en question, mais aussi d'une introspection plus profonde de l'être et la difficulté d'être:

Moi, Shakespeare et Corneille me parlent plus que Marx et Freud, et Arthur Adamov et Armand Gatti plus que Lévi-Strauss et Althusser, sans parler de ce que me parle Bertolt Brecht qui me parle plus que Philippe Sollers, Brecht l'essayiste "animé» par le dramaturge, au discours dont «l'incarné» frappe, et alors immédiatise le sens que ce qui agit soit le rapport parolel corps. Le Carosse d'or, c'est le plus vital de moi qu'il questionne. Et si j'avais raté ma vie? Si mon désir le plus véritable avait été d'être acteur, et que j'y aie renoncé par manque de courage, par lâcheté? Et tout ce que je bois, ne serait-ce point aussi pour jouer grandiosement la comédie que je n'ai pas osée? L'émotion qui m'étreint, la fièvre qui m'arc-boute écorché, l'intensité physique et mentale dès qu'il y a spectacle, coulisses, plateau, scène, passerelles d'éclairages, décors, caméras, loges, représentation qui découvre le réel jusqu'alors incompris, l'expérience crucifixion du trac, et l'entièreté enfin accomplie de qui s'expose dans un jeu où s'exprime extrême son plus vrai, le Narcisse don à tous, potlatch, et le joué fait sens plus que toute théorie 48 .

Dans les cercles étroits de la modernité littéraire, la force de Patrick Straram était justement dans ce perpétuel jeu/action de l'écrivain. Sa production ayant été à la baisse au cours des années 80 , son importance a suivi la courbe. Elle n'avait plus limpact ravageur qu'on lui accordait pendant les années fortes de la contreculture, car elle est maintenant le lot d'une génération vieillissante à laquelle aucune jeune faction ne semble vouloir donner écho, à mesure que l'idéologie occidentale entre un peu plus, chaque jour, dans l'ère du vide.

47 Hobo Québec, op. cit., p. 14.

48 Patrick Straram, Quatre Quatuors en trains qu'amour advienne, p. 57. 